\title{
IMPACTO DE UNA HERRAMIENTA MULTIMEDIAL EN EL PROCESO DE ENSEÑANZA-APRENDIZAJE DE LA HISTORIA EN ELAULA
}

\section{IMPACT OF A MULTIMEDIA TOOL IN THE PROCESS OF LEARNING HISTORY IN THE CLASSROOM}

\author{
Yenny Constanza Murcia Castellanos \\ yennymurcas11@hotmail.com \\ María Lucia Tejedor Estupiñan \\ mariatejedor.16@gmail.com \\ Diana Yaneth Lancheros Cuesta \\ diana.lancheros@campusucc.edu.co
}

\begin{abstract}
Universidad Cooperativa de Colombia, Maestría en Educación, Avenida caracas No 3704 Bogotá (Colombia)
\end{abstract}

Hoy en día las herramientas tecnológicas permiten mejorar los procesos de enseñanza y aprendizaje, el presente artículo muestra los resultados de un proyecto de investigación centrado en definir una estrategia metodológica para el proceso de enseñanza-aprendizaje en la asignatura de Historia de grado $6^{\circ}$ a través de la implementación de las Tecnologías de la Información y la Comunicación -TIC-(multimedia). Para el cumplimiento del objetivo planteado, se desarrollaron los referentes teóricos, así como la elaboración de encuestas diagnósticas y un Pretest, revelador del coeficiente mental tríadico y encuesta de clase magistral y un pos-test. Se implementó una herramienta multimedial que facilitará la comprensión y el recuerdo de conceptos en la materia de Historia de un Colegio en Bogotá. Las niñas luego de la presentación y desarrollo de actividades mediadas por la multimedia, presentaron una mejora significativa en su aprendizaje en la clase de historia.

Palabras claves: TIC, educación, historia, proceso de enseñanza-aprendizaje, MICEA.

Today technological tools can improve the teaching and learning, this article shows the results of a research project focused on defining a methodological strategy for the process of teaching and learning in the course of History of grades 6 to through the implementation of ICT (multimedia) (information and communications technology). To fulfill the stated objective, the theoretical framework, and the development of diagnostic surveys and a pre-test, the triadic revealing IQs and survey lecture and posttest were developed. a multimedia tool that will facilitate understanding and memory of concepts in the field of History of a school in Bogotá was implemented. Girls after the presentation and development of multimedia mediated activities showed significant improvement in their learning in History class.

Keywords: ICT, education, history, teaching-learning process, micea.

$$
-211-
$$

Píxel-Bit. Revista de Medios y Educación. No 50 Enero 2017. ISSN: 1133-8482. e-ISSN: 2171-7966. doi: http://dx.doi.org/10.12795/pixelbit.2017.i50.15 


\section{Introducción.}

En el contexto Colombiano, se presenta una normatividad con respecto a las competencias TIC propuestas por el -MENMinisterio de Educación Nacional que deben desarrollar los docentes dentro del contexto específico de la innovación educativa con uso de TIC. (Ministerio de Educación Nacional, 2013). El desarrollo de estas competencias por los docentes, permiten que se dé un cambio en sus prácticas pedagógicas, lo que influye en los procesos de enseñanza aprendizaje.

El planteamiento del problema, surge de la práctica docente en un Colegio de educación básica y secundaria de Bogotá, en donde se hace evidente que las estudiantes de grado $6^{\circ}$, presentan un desinterés en la asignatura de Historia. Este tipo de aspectos pueden deberse a metodologías y/o temáticas.

Por otro lado el proceso de enseñanza aprendizaje, con mayor frecuencia incluye el uso de TIC, permitiendo al estudiante procesos de adquisición de conocimientos y prácticas para un uso adecuado de estas, principalmente de las plataformas virtuales y redes sociales. (Zárate, 2009). De igual forma las TIC se han ido implementando de forma progresiva en los diferentes contextos de la vida de los colombianos, permeando claramente a la educación.

Ejemplo de lo mencionado anteriormente son los estudios realizados por (Zuluaga \& Cardona., 2012; Díaz, 2012 y Molina, 2009) quienes presentan diversas formas de creación de estrategias metodológicas que contribuyen a la creación de ambientes de enseñanza-aprendizaje mediados por las TIC, mejorando así el interés de los estudiantes por determinados contenidos o asignaturas en el contexto escolar. Ahora bien, el uso de las herramientas TIC en educación debe tener un enfoque que permita la apropiación de elementos tecnológicos con fines educativos, pedagógicos y didácticos, sin dejar de lado la exploración por parte de los estudiantes, de esta manera, se piensa en la apropiación de una herramienta TIC, con el fin de contribuir en el aprendizaje del educando sin que esto implique caer en métodos de enseñanza reproduccionistas.

Los estándares propuestos por la UNESCO (2008) se forman en tres grandes enfoques: 1. Enfoque de nociones básicas de TIC. 2. Enfoque de profundización del conocimiento. 3. Enfoque de generación de conocimiento (UNESCO , 2008). Estos tres enfoques, se articulan a todas las prácticas educativas, es decir que se deben tener en cuenta en la formación de los estudiantes desde primeros años escolares hasta el ámbito universitario, pero también en las formaciones de docentes nuevos en Universidades que contengan las Licenciaturas como carreras y en capacitaciones a docentes en ejercicio.

No se puede pretender de ninguna forma que el cambio educativo se dé en TIC, si no se ha dado una educación en estos tres enfoques presentados por la UNESCO. Intentar un cambio de mentalidad o una apropiación de las TIC sin tener claro en qué consisten, como adaptarla y para qué sirven las TIC es un error en el que se ha caído y que no permite el avance en relación a la implementación de TIC en el campo educacional.

Por lo anterior, el presente artículo muestra los resultados de un proyecto de investigación que implementó una estrategia metodológica para el proceso de enseñanzaaprendizaje en la asignatura de historia de grado $6^{\circ}$ a través de la implementación de las

$$
-212-
$$


TIC y MICEA (metodología centrada en equipos de aprendizaje) (Velandia, 2007).

El artículo muestra en la sección 2 los trabajos que se relacionan con la creación de estrategias para la mejora de la enseñanza dentro del aula mediadas por TIC, con el fin de ser el referente teórico base para el desarrollo de la misma.

\section{Trabajos relacionados}

Realizando un análisis de trabajos en las categorías de educación, TIC y estrategias metodológica se encuentran trabajos como el desarrollado por Ospina, López y Cuesta (2005), quienes implementaron entornos virtuales de aprendizaje bajo la Metodología Interdisciplinaria Centrada en Equipos de Aprendizaje. MICEA», con la investigación se estableció que la metodología se adapta a los procesos educativos, permitiendo tener un referente de éxito en el desarrollo del presente proyecto.

Por otro lado, Ospina (2010) afirma que es importante establecer la articulación de las TIC con el desarrollo de la capacidad de los estudiantes, para aprender a aprender, buscar información de forma selectiva, tener una posición crítica, analítica, argumentativa y propositiva ante la información disponible, establecer ambientes colaborativos y cooperativos de trabajo que les permita socializar y debatir para ayudar a entender y fomentar valores en las TIC como la tolerancia, el respeto por el otro, la solidaridad, la equidad y la justicia.

A nivel internacional algunos países han integrado políticas de educación con TIC en las aulas de clase, por ejemplo en Latinoamérica, se han desarrollado avances en relación con la inclusión de las TIC en la educación, la Comisión Económica para
América Latina y el Caribe -CEPAL-, presenta en el documento "Las tecnologías digitales frente a los desafíos de una educación inclusiva en América Latina Algunos casos de buenas prácticas», en este documento se presentan algunas de las experiencias exitosas de inserción de TIC en educación a lo largo y ancho de América Latina. Adicional en Costa Rica, se desarrolla el Programa Nacional de Informática Educativa (PRONIE-ME-FOD), desde 1988, con el fin de introducir de manera masiva las TIC en la educación pública costarricense. Este programa desde que se creó tenía dos objetivos fundamentales, el primer objetivo tenía que ver con avanzar y lograr la cobertura nacional y el segundo con hacer a los docentes consientes de la necesidad de capacitarse y a sus estudiantes, dado que una computadora por sí sola no representa una transformación pedagógica, es decir que se necesita de una innovación en la práctica educativa.

Algunos países han redactado políticas de apoyo educativo con TIC, por ejemplo Buenos Aires (Argentina), cuenta con el Plan Integral de Educación Digital, creado recientemente en el año 2010, este plan tiene como fin el fin de lograr «un computador por niño», en las instituciones educativas públicas de Buenos Aires, para contribuir con el plan de acceso a las TIC y contribuir al cierre de la brecha digital entre las instituciones educativas públicas y privadas. (Pérez, 2012, 168).

En Uruguay, se desarrolló el plan CEIBAL (Conectividad Educativa de Informática Básica para el Aprendizaje en Línea), basado en un programa universal que pretende otorgarle una laptop a cada niña, niño y docente de todas las escuelas públicas del país. El Plan está basado en el proyecto One

$-213-$

Píxel-Bit. Revista de Medios y Educación. $N^{o} 50$ Enero 2017. ISSN: 1133-8482. e-ISSN: 2171-7966. doi: http://dx.doi.org/10.12795/pixelbit.2017.i50.15 
Laptop Per Child (OLPC), propuesto por Nicholas Negroponte, del Massachusetts Institute of Technology (MIT). Así, el objetivo principal del Plan CEIBAL fue lograr el acceso masivo a las nuevas tecnologías de información y comunicación. Utilizando la escuela como principal medio, su segundo objetivo fue contribuir a la mejora de la calidad educativa mediante la integración de tecnología al aula, al centro escolar y al núcleo familiar cambiando las formas de concebir el proceso de enseñanza aprendizaje en las escuelas públicas de este país.

Con respecto a la enseñanza de la historia Florescano (2002), reconoce en su investigación la historia como un proceso coherente entre los contenidos culturales y los procedimientos y actitudes que informan cada contenido, además integra prácticas de enseñanza con aquellas fuentes que constituyen los fundamentos educativos. En términos generales, la historia se encarga de difundir los valores de la cultura y así poder comprender el sentido de la civilización. En sentido amplio la enseñanza de la historia que es el conducto más adecuado para conocer y reconocer los valores universales que han llevado al ser humano a ser un transmisor eficaz de los valores e identidades nacionales. Por lo tanto, el proceso de aprendizaje de la historia prepara al estudiante para una vida en comunidad, esto teniendo en cuenta el grado de desarrollo del conocimiento propio de cada grupo y, al tiempo, permite realizar enfoques didácticos según las necesidades educativas y capacidades de los educandos. Entonces, dentro de los aportes que da la enseñanza de la historia se puede destacar su contribución al conocimiento y comprensión de las diferentes culturas y sociedades y aprender a valorarlas.
González (2011) afirma que el uso de estos recursos puede distorsionar los fines educativos y pensar que por el simple hecho de usarlos los alumnos adquieren conocimiento. Se trata, en todo, caso de platear estrategias que partan de las necesidades educativas de los propios alumnos. Una pedagogía centrada en el alumno implica, según los especialistas como Paulo Freire y Carl Rogers, (citados por González, 2011), tomar en cuenta el contexto y las circunstancias socio-históricas que viven los alumnos en su tránsito por el bachillerato, pero también desde el paradigma sociocultural Vigotskiano.

Para tener un panorama a nivel nacional, se presentan algunas fuentes secundarias que tienen ciertas coherencias con el desarrollo de estrategias metodológicas y uso de TIC, para contribuir al mejoramiento del proceso de enseñanza aprendizaje.

Por su parte Corredor (2014), muestra el desarrollo de la experiencia pedagógica que tiene en cuenta el modelo Aprendizaje Basado en Proyectos, el modelo de Aprendizaje colaborativo y los estilos propios de los estudiantes para aprender, este proyecto se desarrolla en un contexto rural, en donde el uso de TIC es precario y su acceso es poco y restringido.

De igual forma Grisales (2013), desarrolla una plataforma Moodle en las áreas de matemáticas, ciencias naturales (física, química), humanidades, social, filosofía y ciencias políticas. Para lo cual se capacitaron a los docentes en su manejo, luego se implementó la plataforma y finalmente se evaluó el impacto de la plataforma en docentes y estudiantes. Al finalizar su proyecto, Grisales (2013), concluye que la aplicación de diferentes herramientas $M O O D L E$, le permitieron a los docentes

$$
-214-
$$


dinamizar las clases, involucrar a los estudiantes en los procesos educativos además que permitió una optimización de los recursos y del tiempo utilizado por los docentes en la organización y calificación de trabajos y exámenes.

Finalmente se tiene a Lancheros y Carrillo (2012), quien desarrollo el documento «Modelo adaptativo para la caracterización de dificultades/discapacidades en un ambiente virtual educativo». Las autoras establecen lo siguiente: Las dificultades de aprendizaje en el ámbito educativo, es hoy en día una de las principales causas de deserción estudiantil. Una de las soluciones ha sido el utilizar herramientas pedagógicas y didácticas en ambientes virtuales de aprendizaje que facilitan el proceso educativo y mejoran los procesos cognitivos en los estudiantes. En su artículo presentan los $M D A L S$ (acrónimo de Adaptive Learning Disability Model System), sistema que permite caracterizar una discapacidad o dificultades que un estudiante puede tener en su proceso de aprendizaje. Este sistema se convierte en un apoyo para la construcción de ambientes virtuales con el objetivo de ofrecerle servicios personalizados y/o adaptados a los estudiantes tomando en cuenta su contexto, su perfil y de esta manera apoyarlo en su aprendizaje

Se puede evidenciar cómo han existido relaciones estrechas entre el hombre y la tecnología las cuales son complejas, pero a su vez necesarias. Por una parte, se utiliza la tecnología como herramienta para desarrollar algunas habilidades o capacidades, transformando paulatinamente el entorno.

\section{Material y métodos.}

El proyecto desarrollado se basó en una investigación de corte experimental, de acuerdo con [5], un experimento se lleva a cabo para analizar si una o más variables independientes afectan a una o más variables dependientes y por qué hacen. En un experimento, la variable independiente resulta de interés para el investigador, ya que hipotéticamente será una de las causas que producen el efecto supuesto. Para obtener evidencia de esta supuesta relación causal, el investigador manipula la variable independiente y observa si la dependiente varia o no. Aquí, manipular es sinónimo de hacer variar o asignar distintos valores a la variable independiente.

Se establece que el tipo de investigación establecido para el desarrollo de este proyecto fueron los modelos cuasiexperimentales. El método elegido fue mixto, teniendo los cuantitativos en e desarrollo de encuestas y los test realizados, el método cualitativo se encuentra presente en el análisis de la información recolectada, que da origen a los resultados y la triangulación de los mismos. Partiendo de las variables establecidas así: Variable Dependiente: Historia, y las variables independientes elegidas fueron: Variable 1. TIC, Variable 2. Educación.

La población objetivo (muestra) fue la totalidad de las estudiantes de grado sexto de un colegio privado de Bogotá D.C, en la asignatura de historia en total se trabajó con 65 niñas.

El desarrollo del proyecto se realizó en cinco fases presentadas a continuación: Fase I: En la primera etapa de la investigación se definió el enfoque de la misma, para tener límites claros y definir fuentes de información,

$-215-$

Píxel-Bit. Revista de Medios y Educación. $N^{o} 50$ Enero 2017. ISSN: 1133-8482. e-ISSN: 2171-7966. doi: http://dx.doi.org/10.12795/pixelbit.2017.i50.15 


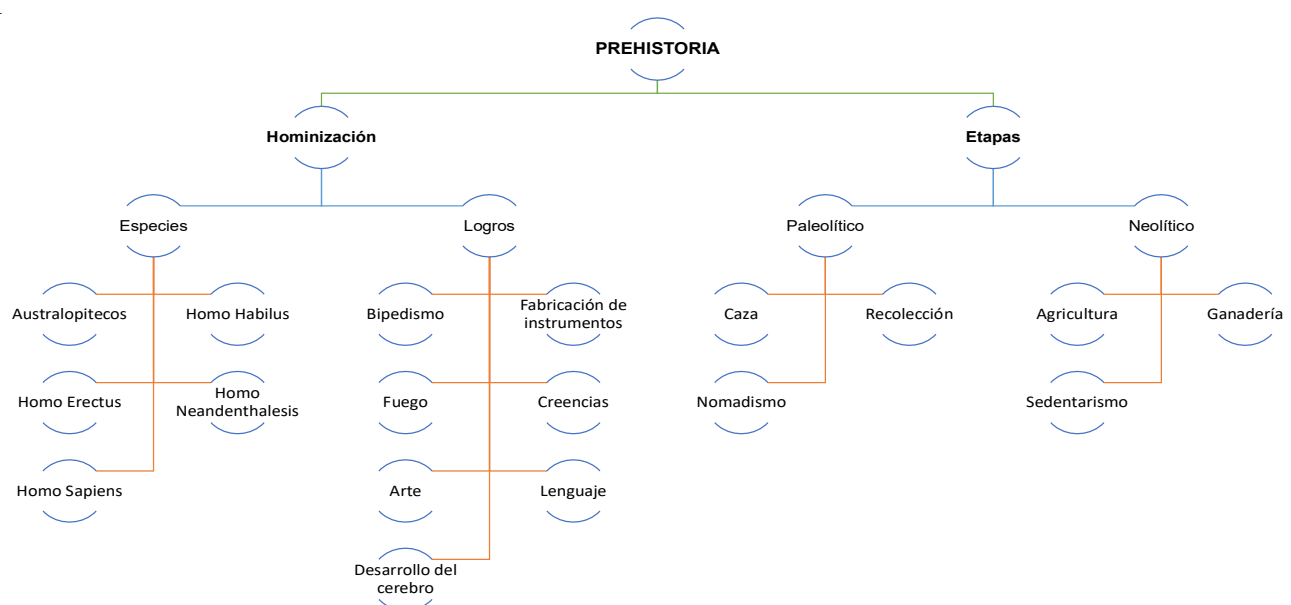

Figura 1. Conceptos de prehistoria de la multimedia. Fuente: Elaboración propia.

la Fase II: Se realizó una encuesta inicial diagnóstica, realizada a los estudiantes del grado $6^{\circ}$ del Colegio en relación con el proceso de enseñanza-aprendizaje de la asignatura de historia. Fase III: Teniendo el diagnóstico y sus resultados, se determinan los componentes de la estrategia metodológica para mejorar el proceso de enseñanza aprendizaje en la asignatura de historia utilizando las TIC. Fase IV: Posteriormente se realiza el diseño, la estrategia metodológica y se realiza su validación en la asignatura de historia con las estudiantes del grado sexto del Colegio y finalmente la Fase V: en donde se presentan los resultados de acuerdo a las variables de análisis seleccionadas para este proyecto, para dar paso a la presentación de

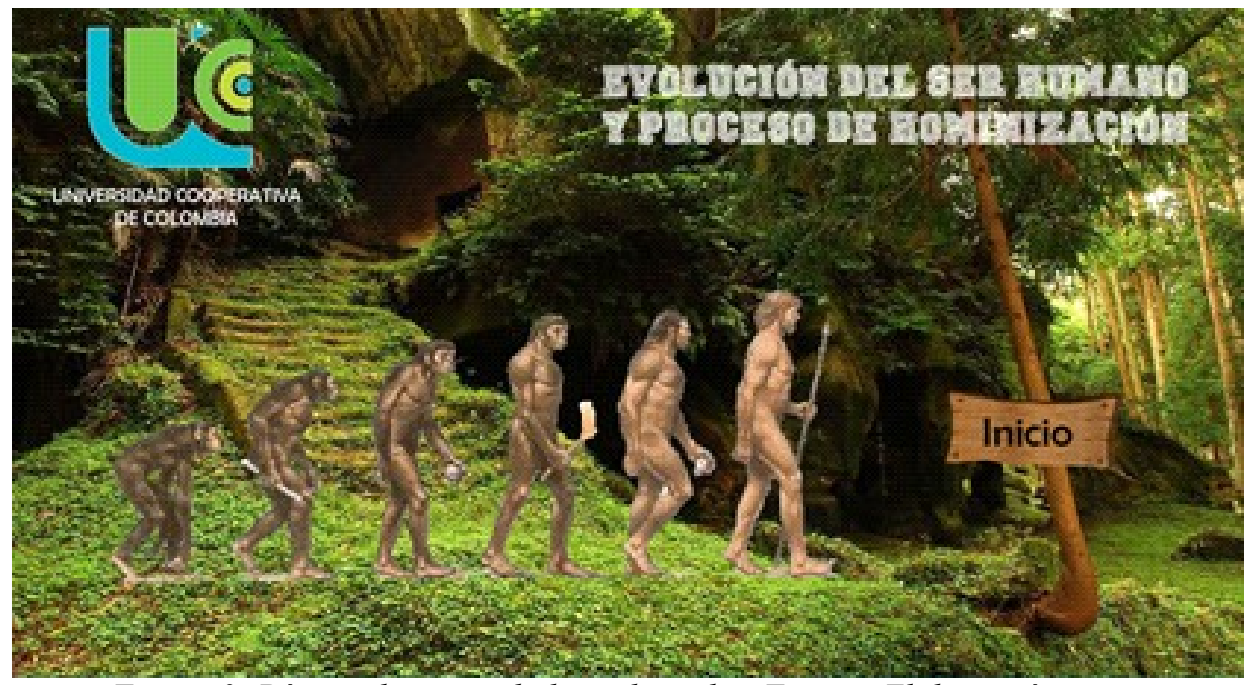

Figura 2. Página de inicio de la multimedia. Fuente: Elaboración propia.

$$
-216-
$$

Píxel-Bit. Revista de Medios y Educación. No 50 Enero 2017. ISSN: 1133-8482. e-ISSN: 2171-7966. doi: http://dx.doi.org/10.12795/pixelbit.2017.i50.15 


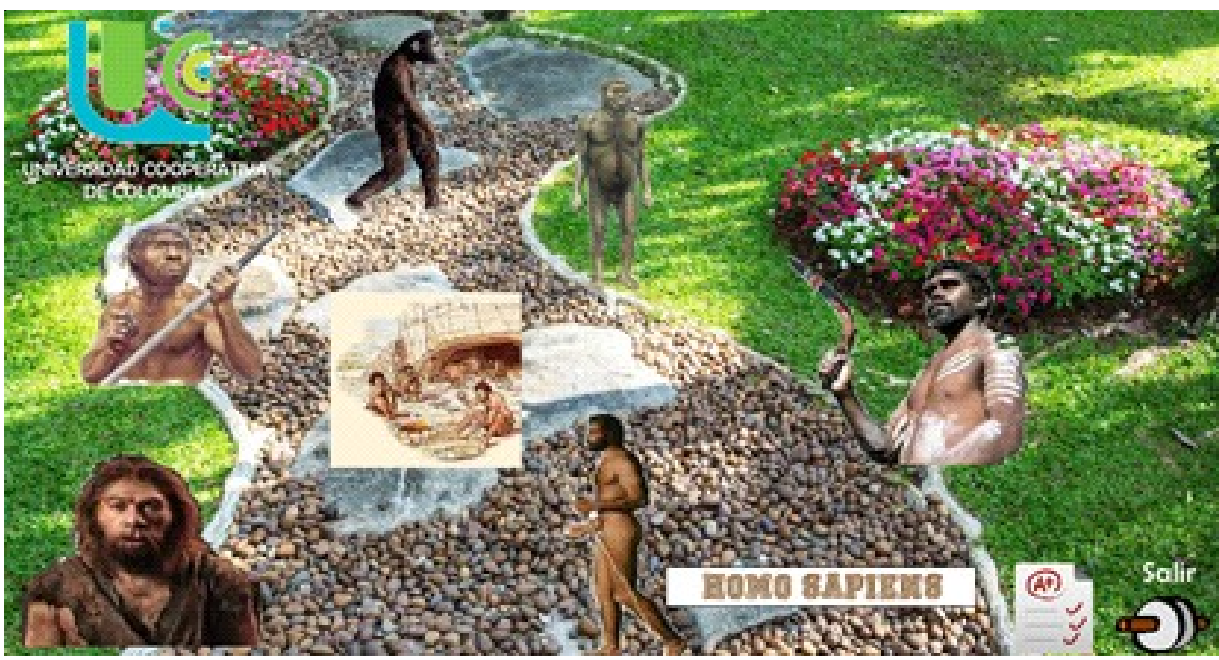

Figura 3. Presentación de contenido de la multimedia. Fuente: Elaboración propia.

conclusiones, de acuerdo con ello, se da cumplimiento a los objetivos general y específicos de la presente investigación.

El instrumento utilizado fue principalmente el test, este se usó de la siguiente manera: el pre-test se elaboró con el fin de lograr la obtención de los datos necesarios para el desarrollo de la multimedia, este test permitió medir las fallas que tienen las niñas en relación con el aprendizaje de contenidos en la asignatura de historia, luego se desarrolló el Test Revelador de Coeficiente Mental Tríadico a las 65 niñas y finalmente se realizó un Post-Test, con el fin de dar validez al desarrollo de la multimedia. La multimedia realizada se basó en los conceptos de prehistoria que se enseñan en el grado $6^{\circ}$, presentados en la Figura 1.

La multimedia desarrollada se presenta a continuación, la página de inicio de esta, los diferentes momentos donde explican la prehistoria y finalmente un Quiz que respondieron las estudiantes, ver Figura 2.

Como se presenta en la Figura 2, las estudiantes, pueden dar click en las imágenes y conocer más acerca de cada uno de los homos. La Figura 3 muestra un ejemplo de la presentación de la herramienta en cada una de las opciones.

Como se observa en la Figura 4, al interior de cada imagen se encuentra un poco de historia acerca de la especie, en la siguiente se presenta el Homo Sapiens.

Al observar todas las informaciones, se realizó el Quiz de manera individual, de esta manera se evalúan los conocimientos adquiridos por medio de la multimedia. Un ejemplo de cómo la se realiza la evaluación en la herramienta se muestra en la Figura 5.

El Post Test (Quiz) contó con 15 preguntas, se diseñó con el fin de lograr visualizar el cambio en la adquisición de conocimientos por parte de las niñas, con el fin de establecer si el desarrollo de multimedias, permite que el proceso de Enseñanza aprendizaje mejore.

\section{Resultados}

La presentación de resultados, de esta propuesta se desarrolló con base en el uso 


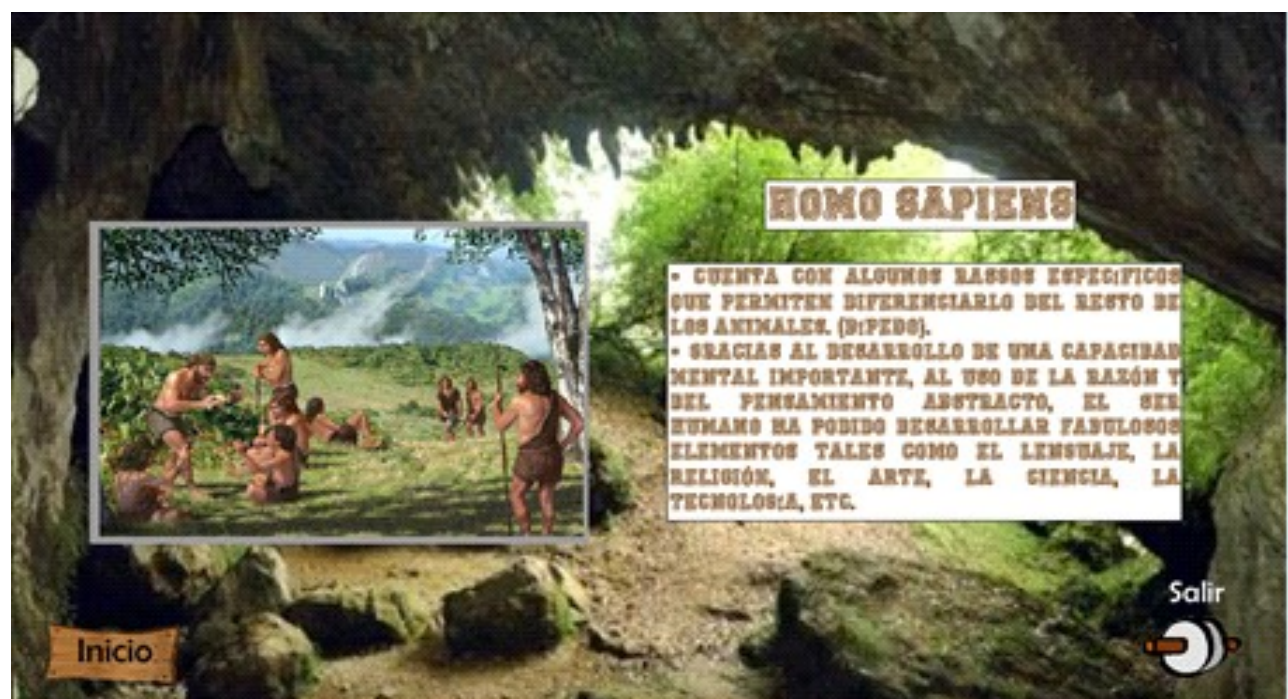

Figura 4. Contenido de cada imagen. Fuente: Elaboración propia.

de MICEA, concretamente del aula dinámica, la motivación, 2) la ubicación, 3) la adquisición, la cual de acuerdo con Velandia, (2005) se 4) la asimilación, 5) la síntesis, 6) la creatividad, compone de diez momentos que conforman 7) el compromiso, 8) el desempeño, 9) la el trabajo del docente, desde la estrategia del evaluación y 10) la reorientación. Su objetivo aula dinámica planteada en MICEA, son: 1) es hacer una retroalimentación al estudiante

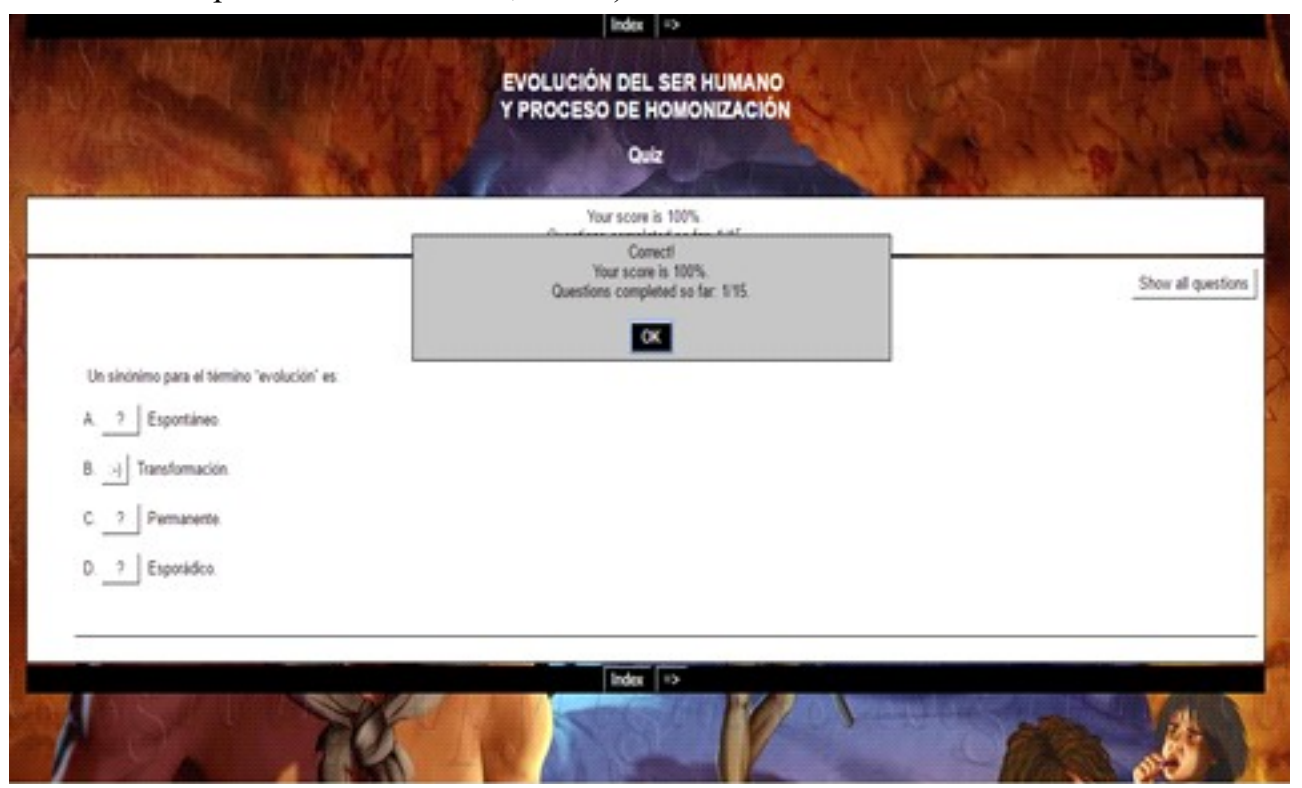

Figura 5. Evaluación realizada en la plataforma. Fuente: Elaboración propia.

$$
-218-
$$

PIXEL Píxel-Bit. Revista de Medios y Educación. No 50 Enero 2017. ISSN: 1133-8482. e-ISSN: 2171-7966. doi: http://dx.doi.org/10.12795/pixelbit.2017.i50.15 


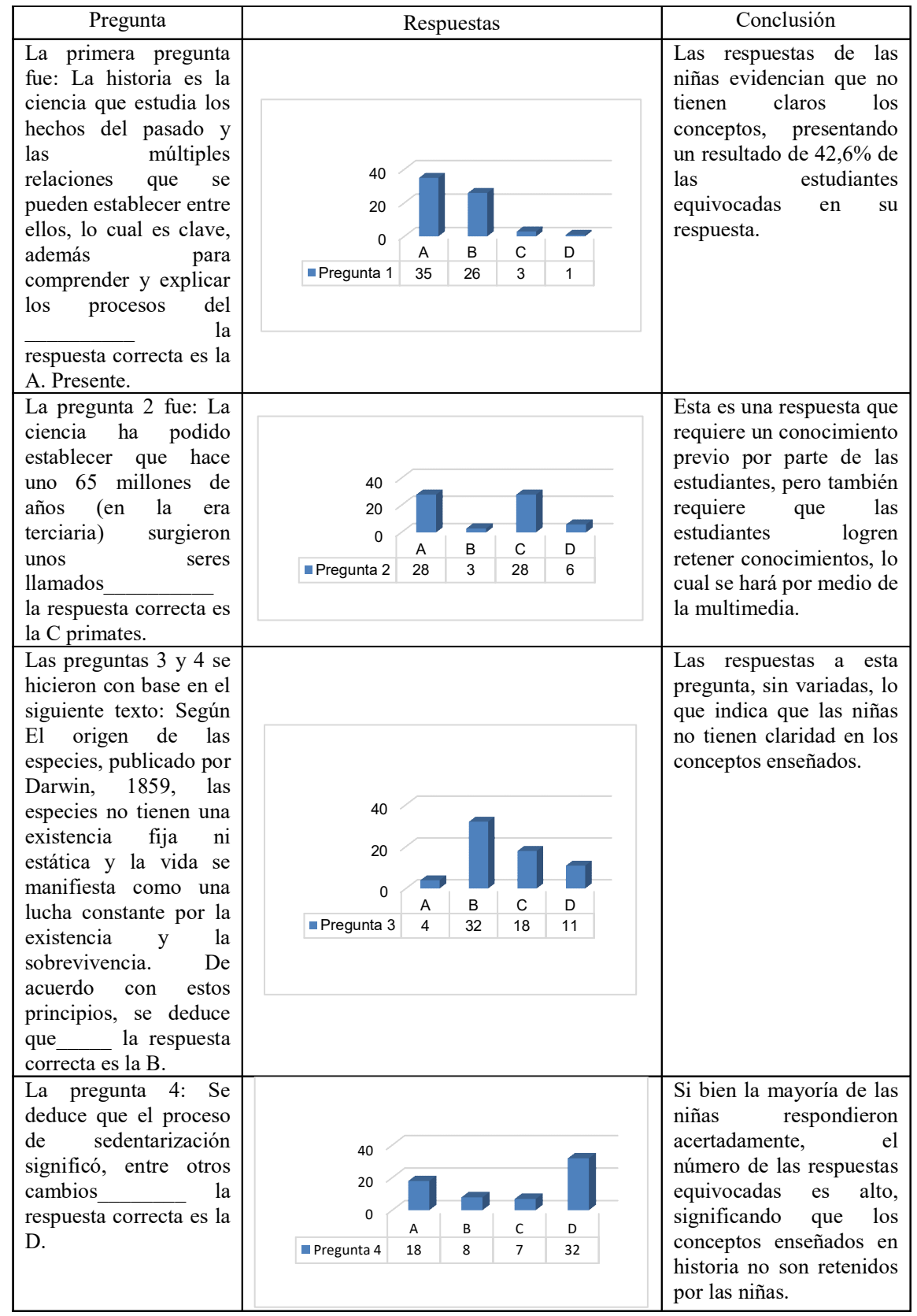

Tabla 1. Resultados Pre test Fuente: Elaboración propia.

Píxel-Bit. Revista de Medios y Educación. No 50 Enero 2017. ISSN: 1133-8482. e-ISSN: 2171-7966. doi: http://dx.doi.org/10.12795/pixelbit.2017.i50.15 


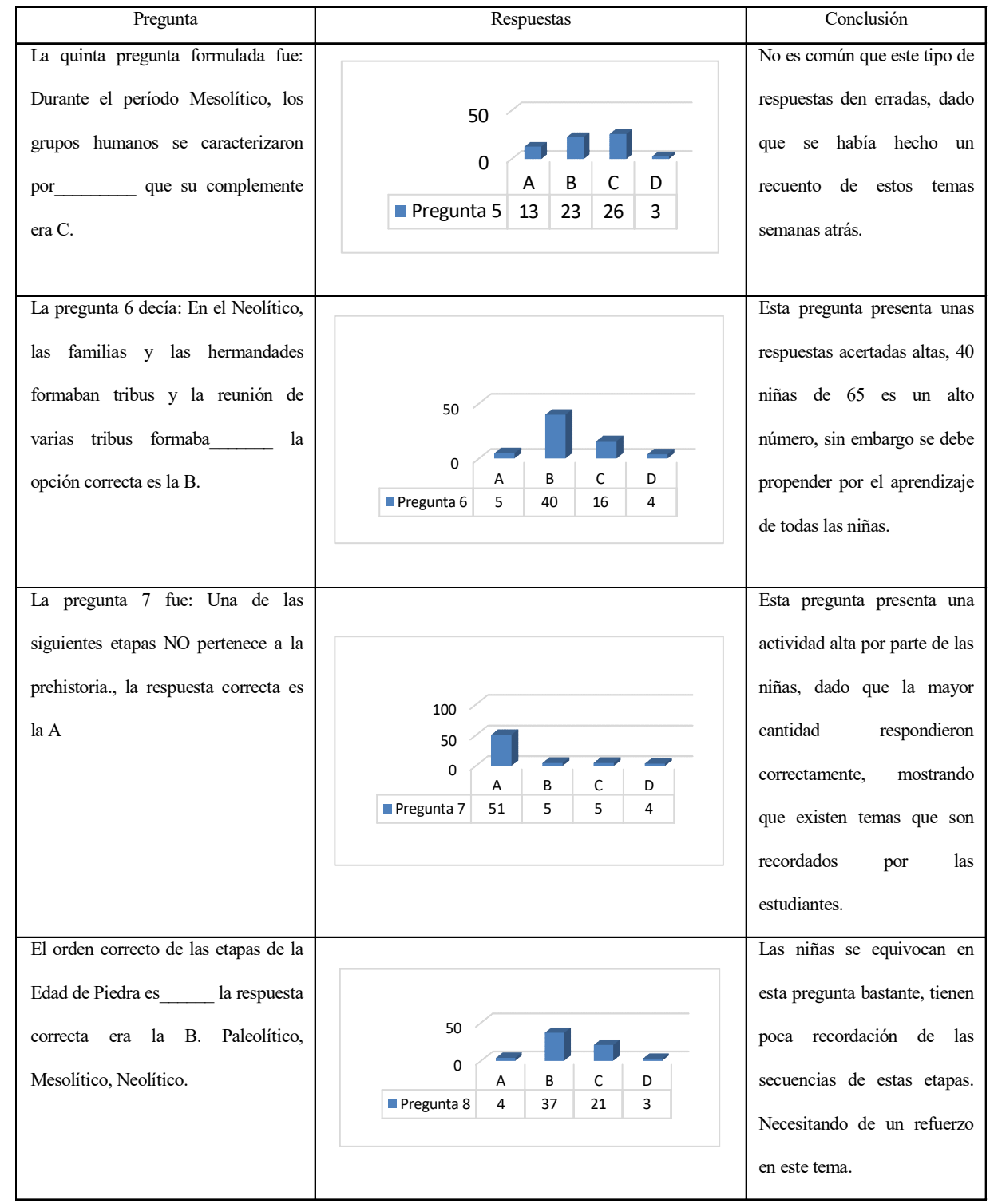

Tabla 1. Resultados Pre test Fuente: Elaboración propia. (continuación)

que le permita reforzar los logros alcanzados y llenar los vacíos encontrados.

En primer lugar, un Pre Test, mediante el cual se define la motivación actual de las estudiantes para el desarrollo del proceso de enseñanza-aprendizaje de la asignatura de historia, definiendo así se logra la ubicación de los problemas de adquisición de conocimientos y la asimilación de los mismos.

$$
-220-
$$

Píxel-Bit. Revista de Medios y Educación. No 50 Enero 2017. ISSN: 1133-8482. e-ISSN: 2171-7966. doi: http://dx.doi.org/10.12795/pixelbit.2017.i50.15 
COCIENTE MENTAL TRIADICO - $6^{\circ}$

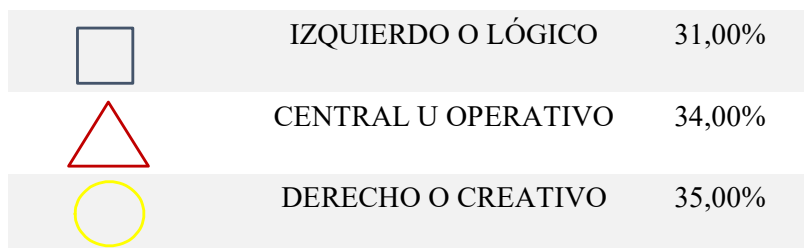

Tabla 2. Resultados Test Revelador del Cociente Mental Tríadico.

Fuente: Elaboración propia.

El momento 5, se realizó mediante el análisis de los resultados de tres actividades: 1 . PreTest, 2. Realización del Test Revelador del coeficiente mental Tríadico. 3. Primera encuesta diagnóstica para establecer las opiniones de las niñas en relación al desarrollo de las clases magistrales de historia. Los momentos 6 a 10 se realizaron mediante el diseño, desarrollo e implementación de la multimedia diseñada. La tabla 1 muestra en detalle las preguntas realizadas y un análisis de las respuestas.

Las respuestas presentadas en la tabla 12 , permiten observar que las niñas no logran retener por largos periodos de tiempo los contenidos de la asignatura de historia, lo que necesariamente impacta las calificaciones de las niñas y genera problemas para que ellas logran tener una continuidad en los contenidos enseñados. Se requiere que las estudiantes, puedan tener claros los contenidos y recordarlos a largo plazo.

Las mayores dificultades presentadas, tienen que ver con la recordación de secuencias, es decir la secuencia de las etapas de la edad de piedra y las de los homos, así como la continuidad de los hechos en la línea del tiempo, tal como se muestra en la pregunta 8 .

Se realizó la segunda prueba diagnóstica denominada Revelador del coeficiente mental Tríadico, los resultados de las 65 niñas testadas muestran que la mayoría tiene más desarrollado el derecho o creativo con el $35 \%$ del total, seguido del central operativo con $34 \%$ y el izquierdo Lógico con $31 \%$. Estos resultados se evidencian en la Tabla 2 .

Finalmente, en la tabla 3 se pueden observar los resultados del pos-test, que evidencian el impacto de la multimedia mejorando la adquisición de conocimientos en el de Historia por parte de las estudiantes de grado sexto.

Los resultados demuestran que la implementación de herramientas TIC en el proceso de enseñanza-aprendizaje, mejoró los resultados de las niñas del grado $6^{\circ}$, en este sentido, se concluye que el desarrollo de estrategias metodológicas mejora los procesos de enseñanza-aprendizaje, por medio del uso de TIC, por tanto, estos aportes deberían ser realizados con mayor frecuencia en las instituciones escolares.

\section{Discusión}

Los resultados del desarrollo de este proyecto muestran que antes del desarrollo de las actividades con la multimedia los conocimientos no se retenían con facilidad y las niñas expresan que la clase era poco llamativa, generando en ellas pereza para el

$-221-$

Píxel-Bit. Revista de Medios y Educación. $N^{o} 50$ Enero 2017. ISSN: 1133-8482. e-ISSN: 2171-7966. doi: http://dx.doi.org/10.12795/pixelbit.2017.i50.15 


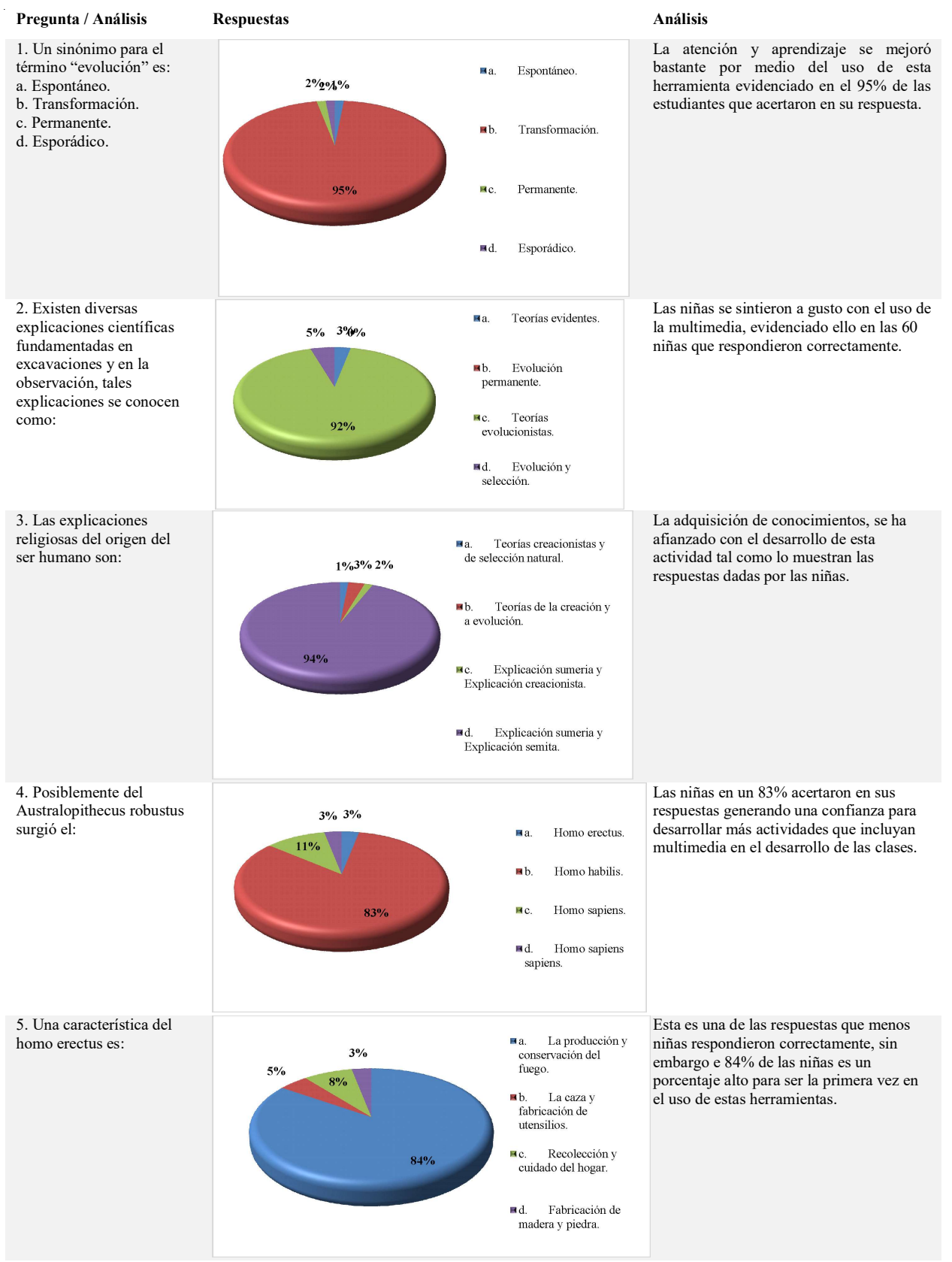

Tabla 3. Resultados Post Test. Fuente: Elaboración propia.

$-222-$

Píxel-Bit. Revista de Medios y Educación. $N^{o} 50$ Enero 2017. ISSN: 1133-8482. e-ISSN: 2171-7966. doi: http://dx.doi.org/10.12795/pixelbit.2017.i50.15 


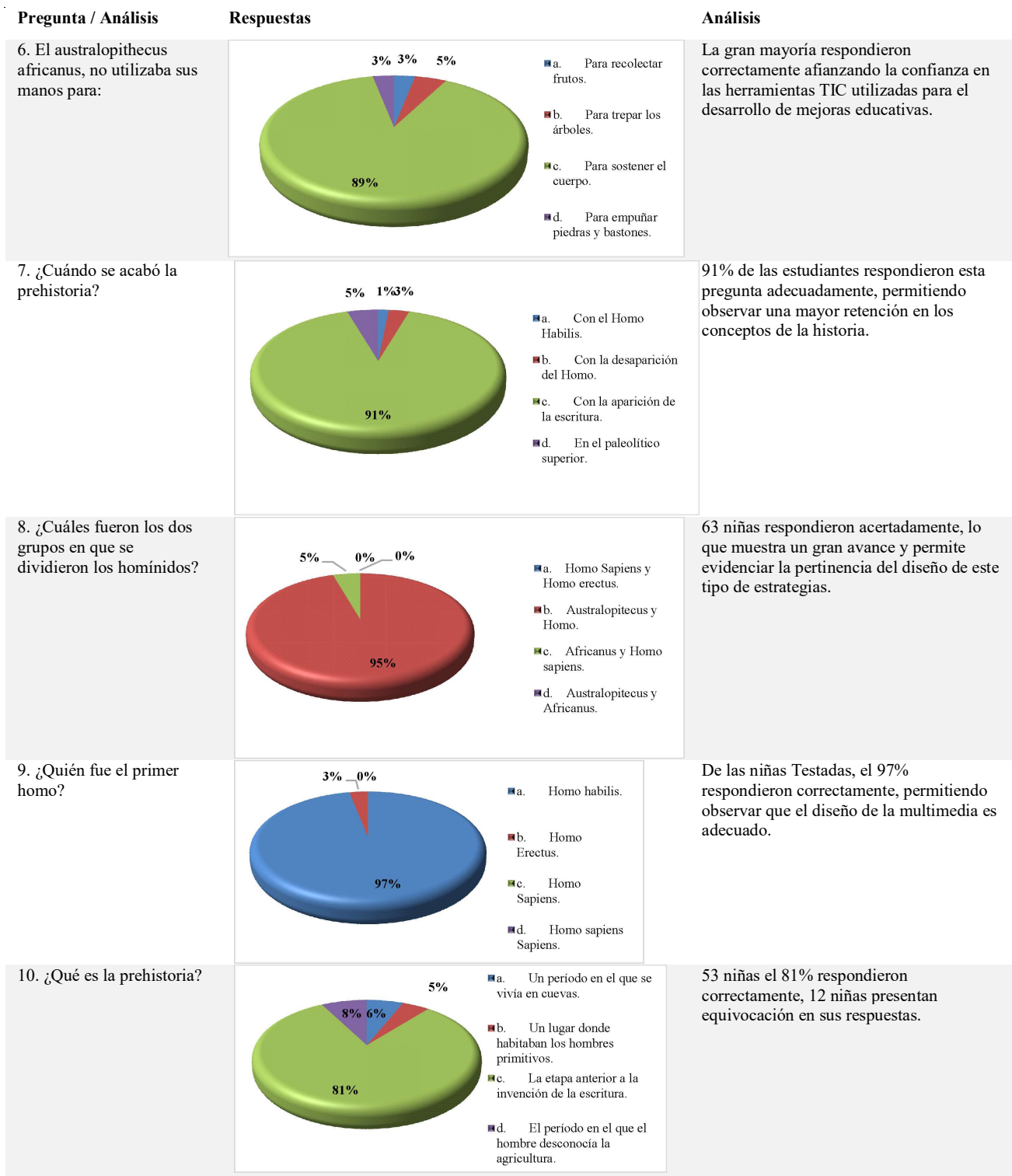

Tabla 3. Resultados Post Test. Fuente: Elaboración propia. (continuación)

$-223-$

Píxel-Bit. Revista de Medios y Educación. No 50 Enero 2017. ISSN: 1133-8482. PIXEI e-ISSN: 2171-7966. doi: http://dx.doi.org/10.12795/pixelbit.2017.i50.15 


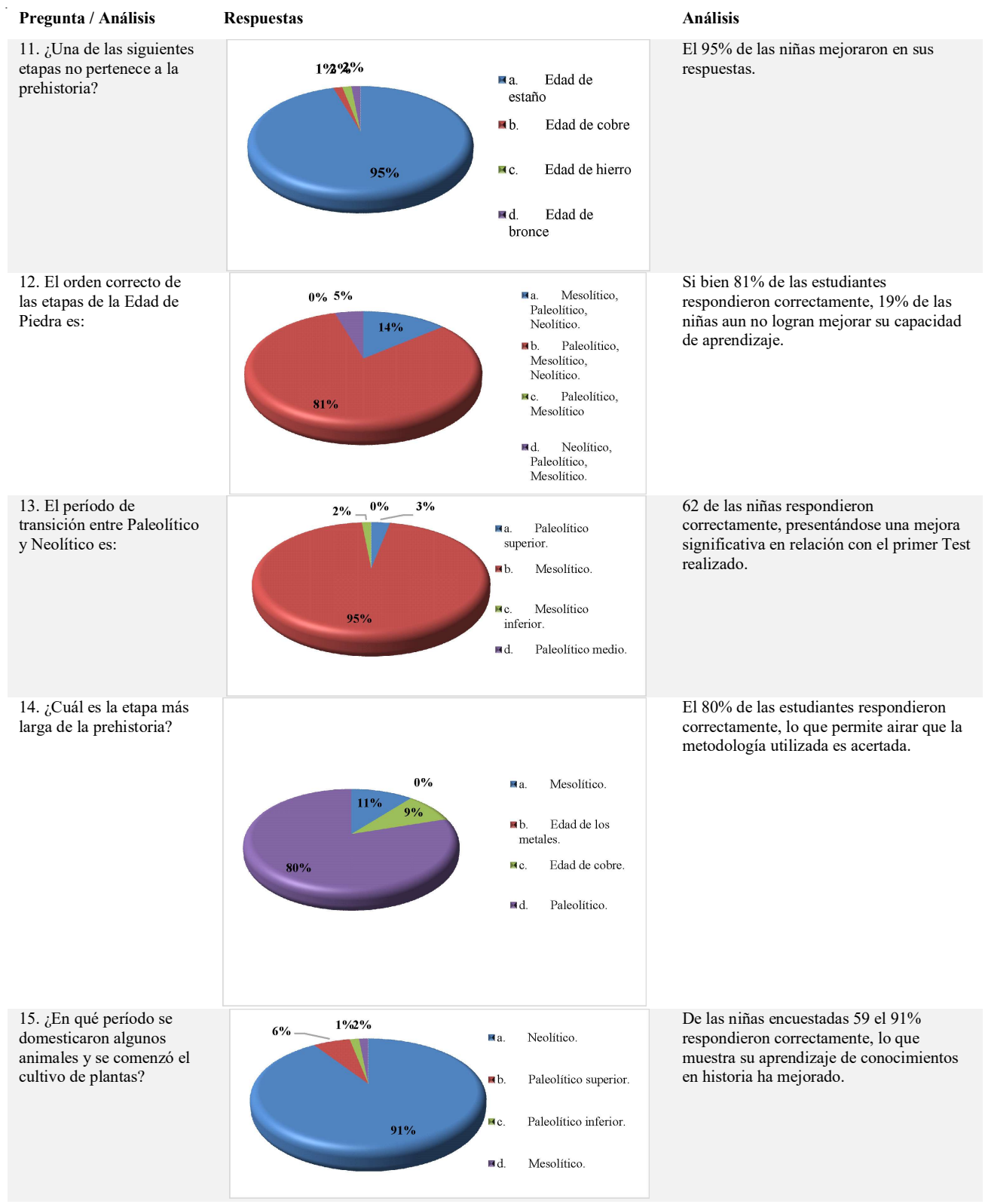

Tabla 3. Resultados Post Test. Fuente: Elaboración propia. (continuación)

$$
-224-
$$

Píxel-Bit. Revista de Medios y Educación. No 50 Enero 2017. ISSN: 1133-8482. e-ISSN: 2171-7966. doi: http://dx.doi.org/10.12795/pixelbit.2017.i50.15 
desarrollo de actividades, lo que no dejaba que tuvieran claros los conceptos enseñados. Esto se hace evidente en el PreTest, donde un alto número, respondía equivocadamente, en especial en las preguntas donde las respuestas eran parecidas por ejemplo la pregunta dos donde dos respuestas la correcta y una equivocada obtuvieron igual cantidad de respuestas.

Como se observó en el estado del arte y el marco teórico, la mayoría de propuestas expuestas, se realizan unidas a las TIC. Tal como lo expresa López (2005) Las Ciencias Sociales enfrentan en la actualidad el desafío de incorporar nuevas dimensiones de la vida de los seres humanos, haciéndose necesario el aprendizaje del pasado para comprender el presente y predecir factores del futuro, La enseñanza de la historia es importante por ser la memoria de la humanidad que nos introduce en la evolución de la civilización y los logros del género humano Pratz y Santacana (2011).

La UNESCO (2008) establece que los docentes deben desarrollas determinadas competencias TIC que influyen en el proceso de enseñanza- aprendizaje las cuales son: Guiar la práctica docente en relación al uso de las TIC en el aula, Enfoque de nociones básicas de TIC Enfoque de profundización del conocimiento Enfoque de generación de conocimiento. En la asignatura de historia, las niñas tienen la posibilidad de por medio de estas competencias de los docentes, adquirir por un lado conocimientos de tipo lógico y por otro desarrollar habilidades en el uso de TIC.

\section{Conclusiones}

Para los docentes de historia, se hace necesario que se desarrollen medios educativos y didácticos que permitan que los estudiantes, sin importar su condición social, física o mental, logren la adquisición de conocimientos para que en un futuro puedan responder con buenos resultados ante las pruebas del estado, permitiéndoles su entrada a una Institución de Educación Superior de calidad y le conviertan en una persona con un futuro promisorio. El desarrollo de proyectos de este corte, permiten tener una visión de cómo los estudiantes perciben el proceso enseñanza aprendizaje por un lado y por otro, como se desarrolla la práctica docente y cuáles son los cambios necesarios.

\section{Referencias.}

Alonso, M. G., \& Santos, T. (2012). La experiencia del programa multimedia Uantakua en México. En G. Sunkel, \& D. Trucco, Las tecnologías digitales frente a los desafios de una educación inclusiva en América Latina. Algunos casos de buenas prácticas (págs. 147-185). Santiago de Chile: CEPAL.

Bonilla, J. (2003). Politicas nacionales de educación y nuevas tecnologías: el caso de Uruguay. Buenos Aires,: IIPE-UNESCO, $117-$ 129.

Cabero, J. (2004). Las TICs como elementos para la flexibilización de los espacios educativos: retos y dificultades. Comunicación y Pedagogía. España, 13-19.

Cano, J. (2002). La Ecoescuela: una fórmula para la Educación Ambiental. Sevilla: Consejería de Educación y Ciencia de la Junta de Andalucía.

Corredor, N. A. (2014). Estrategias de Aprendizaje Basado en Proyectos con uso de TIC aplicada en Ciencias Sociales. Bogotá: Universidad Autónima de Bucaramanga-Tecnológio. 
Cyranek, G. (2009). En el camino del plan CEIBAL. Referencias para padres $y$ educadores. . Montevideo-Uruguay: UNESCO.

Díaz, S. (2012). Diseño e implementación de una estrategia didáctica para la enseñanza aprendizaje de la Tabla Periódica y sus propiedades en el grado octavo utilizando las nuevas tecnologías TICs: Estudio de caso en la Institución Asia Ignaciana grupo 8-5. Tesis. Madellín: Universidad Nacional de Colombia. Sede Medellín.

Florescano, E. (2002). Para qué enseñar y estudiar historia. Revista de estudios históricos. México, $\mathrm{N}^{\circ} 35$, enero-junio, 135146.

Fonseca, A. (2009). El maestro en el contexto de la cultura digital. Aula Urbana No. 74, , 4-5.

González, S. (2011). Aplicaciones Didácticas de las Nuevas Tecnologías en la Enseñanza de la Historia Crítica en el CCH. Revista del Colegio de Ciencias y Humanidades Para el Bachillerato. Mexico, Eutopía junio 2011 número extraordinario, 193 198.

Grisales, C. A. (2013). Implementación de la plataforma Moodle en la Institución Educativa Luis López de Mesa. Tesis o trabajo de investigación presentado como requisito parcial para optar al título de: Magister en Enseñanza de las Ciencias Exactas y Naturales. Medellín, Colombia: Universidad Nacional de Colombia Facultad de ciencias Exactas y Naturales.

Jaramillo, P. E., \& Ruiz, M. (2009). A case of integration of Information and Communication Technologies that does not add value to learning. Revista latinoamericana de Ciencias Sociales, 1521.
Lancheros, D., \& Carrillo, A. (2012). Modelo adaptativo para la caracterización de dificultades/discapacidades en un ambiente virtual educativo. Dyna, año 79, Nro. 175 , 52-61.

Linares, S. (1994). Los Aprendices y las Matemáticas: el proceso de aprendizaje matemático. En G. Hoz, La Enseñanza de las Matemáticas en la Educación Intermedia (págs. 183-223). Madrid, España: Rialp.

López, E. (2005). Eduteka. Obtenido de Integración de las TIC en Ciencias Sociales. : http://www.eduteka.org/Editorial21.php

Lugo, M. T. (2008). Las políticas TIC en la educación de América Latina. Tendencias y Experiencias. Revista Fuentes, 52-68.

Mateu, E., \& Casal, J. (2003). Tamaño De La Muestra. Universitat Autònoma de Barcelona, 08193-Bellaterra, Barcelona.

MEN, M. d. (2006). Plan nacional decenal de educación 2006 -2016. Pacto social por la educación. Bogotá: Ministerio de Educación Nacional.

Ministerio de Educación Nacional. (2008). Apropiación de TIC en el desarrollo profesional docente. [citado junio 20 2016]. Disponible en: http:// www.colombiaaprende.edu.co/html/ p rod u c tos / $1685 /$ a r t i cle s 312270_Competencias_doc.pdf

Ministerio de Educación Nacional. (2013). Competencias TIC para el desarrollo profesional docente. Bogotá: Imprenta Nacional

Molano, A. (24 de Octubre de 2013). Merani, un verdadero ejemplo de TIC en el aula. Obtenido de Colombia Digital : http:// colombiadigital.net/actualidad/experiencias/ item/5880-merani-un-verdadero-ejemplo-detic-en-el-aula.html

$$
-226-
$$


Molina, R. (2009). La cultura digital de los jóvenes. Del anonimato al show de la intimidad. Aula Urbana No. 74, 18-19.

Mora, C. V. (2001). Metodología Interdisciplinaria. Bogotá: Universidad Cooperativa de Colombia, 54-59.

Moreira, M. (2010). El proceso de integración y uso pedagígico de las TIC en los Centros Educativos. Un estudio de casos. Revista de Educación, 352. Mayo-Agosto 2010,77-97.

Navarrete, C. G. (2010). Análisis comparativo de metodologías de aprendizaje colaborativo, Jigsaw y Aprendizaje Basado en Problemas, haciendo uso de objetos de aprendizaje reutilizables, para el aprendizaje de la geometría, en alumnos de primero medio. Chile: Universidad de Chile.

Ospina, C., López, M., \& Cuesta, A. (2005). Plataforma tecnológica Evas-M. Entornos virtuales de aprendizaje bajo la Metodología Interdisciplinaria Centrada en Equipos de Aprendizaje. Virtual Educa, 1-20.

Ospina, W. (2010). Preguntas para una nueva educación. Discurso de apertura. Congreso iberoamericano Metas 2021, . Buenos Aires Argentina. pag. 12-13.

Pérez, P. (2012). TIC, educación y diversidad: el Plan Integral de Educación Digital de la Ciudad de Buenos Aires. En G. Sunkel, \& D. Trucco, Las tecnologías digitales frente a los desafios de una educación inclusiva en América Latina. Algunos casos de buenas prácticas (págs. 165-186). Santiago de Chile: CEPAL.

Prats, J. \& Santacana, J. (2011). ¿Por qué y para qué enseñar historia?. En J. Prats (ed.), Didáctica de la Geografía y la Historia (pp. 13-29). Barcelona: Graó.

Rodríguez, C., \& Hine, N. (2009). Media as Medium in Colombian Education: How Media and ICT are Changing Communication and the Curriculum in the School. Colombian Applied Linguistics Journal. $N^{\circ} 11$. Junio/ Dicienbre. Bogotá, 32-45.

Rueda, R. (2009). Cibercultura y educación: mundos que habitamos, mundos que creamos. Aula Urbana No. 74, 16.17.

Sánchez, M. E., Venegas, C. A., \& Dalmau, E. A. (2010). Los software educativos como herramientas didácticas mediadoras del aprendizaje. Revista de la Universidad de la Salle, 215-232.

Sánchez, V. (1994). Currículo y Educación Matemática. En G. Hoz, La Enseñanza de las Matemáticas en la Educación Intermedia (págs. 19-142). Madrid, España: Rialp.

Sunkel, G., \& Trucco, D. (2012). Las tecnologías digitales frente a los desafios de una educación inclusiva en América Latina Algunos casos de buenas prácticas. Santiago de Chile: Comisión Económica para América Latina y el Caribe (CEPAL).

Tello, J. (2003). Competencia informática del profesorado de Infantil y Primaria en Huelva y su incidencia en la práctica docente. Tesis de licenciatura. Mexico: Prinles.

UNESCO . (8 de Enero de 2008). Estándares de competencias en tic para docentes. [citado junio 20 2016]. Disponible en: http:// portal.unes co.org/es/ev.ph p URL_ID $=41553 \& U R L \_D O=D O \_T O P I$ C\&URL_SECTION $=201 . \mathrm{html}$ http:// cst.unesco-ci.org/sites/projects/cst/ default.aspx

Uribe, J. J. (Agosto de 1998). ¿Para qué la historia? Revista de Estudios Socales, Vol 01, 44-49.

Velandia, M. (2005). Modelo pedagógico con fundamentos en cibernética social. Bogotá: Universidad Cooperativa de Colombia. 
Velandia, C. (2007). Metodología Interdisciplinaria Centrada en Equipos de Aprendizaje MICEA para la Educación Virtual. Bogotá: Universidad Cooperativa de Colombia.

Vesga, L. d., \& Vesga, J. d. (2012). Teachers towards communication and information technollogies intrution in school scenario. Revista de educación, vol.14 no.19, 10-19.

Xu, D., Wang, H., \& Wang, M. (2010). A conceptual model of personalized virtual learning environments. Educación y Pedagogía. Universidad de los Andes. Vol 29, 525-534.

Zárate, A. (2009). Los actores de la educación frente a las TIC's. Aula Urbana No.74, 3-4.

Zuluaga, D. A., \& Cardona., J. A. (2012). Propuesta metodológica para el currículo del área de español, grado noveno del colegio nacional académico, en un ambiente de aprendizaje mediado por las TIC. tesis para optar por el título en: Licenciatura en Comunicación e Informática Educativa. Pereira.: Universidad Tecnológica de Pereira.

Fecha de recepción: 24-04-2016

Fecha de evaluación: 01-09-2016

Fecha de aceptación: 01-10-2016

$-228-$

Píxel-Bit. Revista de Medios y Educación. No 50 Enero 2017. ISSN: 1133-8482. e-ISSN: 2171-7966. doi: http://dx.doi.org/10.12795/pixelbit.2017.i50.15 